\title{
On Hamiltonian formulation of the Einstein-Hilbert action in two dimensions
}

\author{
N. Kiriushcheva and S.V. Kuzmin* \\ Department of Applied Mathematics, \\ University of Western Ontario, London, N6A $5 B^{\text {r7 Canada }}$
}

(Dated: October 8, 2018)

\begin{abstract}
It is shown that the well-known triviality of the Einstein field equations in two dimensions is not a sufficient condition for the Einstein-Hilbert action to be a total divergence, if the general covariance is to be preserved, that is, a coordinate system is not fixed. Consequently, a Hamiltonian formulation is possible without any modification of the two dimensional Einstein-Hilbert action. We find the resulting constraints and the corresponding gauge transfromations of the metric tensor. Keywords: Hamiltonian formulation, Einstein-Hilbert action, two dimensions.
\end{abstract}

PACS numbers: 11.10.Ef

*Electronic address: nkiriush@uwo.ca, skuzmin@uwo.ca 
In this letter we reconsider the canonical formulation of the two dimensional EinsteinHilbert (EH) action. It is generally believed that the Einstein Lagrangian is a total derivative in two dimensions $(2 D)$ [1, 2] and that its canonical formulation cannot be constructed. (In 3] it is stated that "the canonical formalism breaks down" and in [4] we find that in two spacetime dimensions EH action is meaningless and its modification is needed. It is true that if the Lagrangian $\sqrt{-g} R$ is a total divergence and it is dropped, then the remaining cosmological term in the Lagrangian $-\Lambda \sqrt{-g}$ contains no derivatives and the canonical momenta cannot be defined [5].) However, this statement deserves a closer examination as it seems to contradict some general principles. The Einstein-Hilbert action is valid in any dimension and, of course, can have specific behavior in a particular dimension, but the absence of a canonical formulation would be similar to the claim that an action has no equations of motion in some dimension which is incorrect (equations of motion can be trivial, but they do exist). In such a situation it is natural to expect that the Hamiltonian formulation of a theory can be trivial, leading to zero degrees of freedom, but it does exist. Moreover, if an action is a total derivative (in covariant form) in a particular dimension it has to be a total derivative in all dimensions which is not the case for the Einstein-Hilbert action.

The source of such belief is originated from the well-known fact ([6], [7]) that the Einstein equations

$$
R_{\mu \nu}-\frac{1}{2} g_{\mu \nu} R=0
$$

are trivial in $2 D$. This is easy to demonstrate using the fact that only one component of Riemann tensor is independent in $2 D[6]$. It can also be shown by straightforward variation of the EH action (we use the signature $(+,-,-, \ldots)$ ):

$$
S_{E H}=\int d^{D} x \sqrt{(-1)^{D-1}} g g^{\mu \nu} R_{\mu \nu},
$$

with respect to metric tensor $g_{\mu \nu}$. (Here $g=\operatorname{det}\left(g_{\mu \nu}\right), R_{\mu \nu}$ is Ricci tensor $R_{\mu \nu}=\Gamma_{\mu \nu, \lambda}^{\lambda}-$ $\Gamma_{\mu \lambda, \nu}^{\lambda}+\Gamma_{\sigma \lambda}^{\lambda} \Gamma_{\mu \nu}^{\sigma}-\Gamma_{\sigma \mu}^{\lambda} \Gamma_{\nu \lambda}^{\sigma}$ and $\Gamma_{\mu \nu}^{\sigma}$ is Christoffel symbol $\left.\Gamma_{\mu \nu}^{\sigma}=\frac{1}{2} g^{\lambda \sigma}\left(g_{\mu \sigma, \nu}+g_{\nu \sigma, \mu}-q_{\mu \nu, \sigma}\right).\right)$

However, the conjecture that "correspondingly the EH action is a surface term" [8], based on the fact that the equations of motion are trivial, is not obvious and has to be checked. The triviality of equations of motion is a necessary condition for the Lagrangian to be a total divergence but not a sufficient one. We shall now show that the EH action in two dimensions provides a counter example. 
The extraction of the terms in the $\mathrm{EH}$ action that can be cast into a total divergence is well-known in any dimension [6]. This separation has been used in attempts to devise Hamiltonian formulation of General Relativity (GR) 9], 10]. We can write the action of (21) as

$$
S_{E H}=\int d^{D} x L_{\Gamma \Gamma}-\int d^{D} x\left(V^{\alpha}\right)_{, \alpha}
$$

where the "Gamma-Gamma" part is

$$
L_{\Gamma \Gamma}=\sqrt{(-1)^{D-1} g} g^{\mu \nu}\left(\Gamma_{\sigma \mu}^{\lambda} \Gamma_{\nu \lambda}^{\sigma}-\Gamma_{\sigma \lambda}^{\lambda} \Gamma_{\mu \nu}^{\sigma}\right)
$$

and $V^{\alpha}$ is

$$
V^{\alpha}=\sqrt{(-1)^{D-1} g}\left(g^{\alpha \mu} \Gamma_{\mu \nu}^{\nu}-g^{\mu \nu} \Gamma_{\mu \nu}^{\alpha}\right)=\sqrt{(-1)^{D-1} g}\left[g^{\alpha \mu} g^{\nu \beta}\left(g_{\nu \beta, \mu}-g_{\nu \mu, \beta}\right)\right] .
$$

After extracting the total divergence, the Jackiw conjecture [8] has to lead to zero GammaGamma part in the $2 D$ action. We can, however, show that this is incorrect. We express the Gamma-Gamma term in the form (see Eq. (2) of [9] or Eq. (8) of [10])

$$
L_{\Gamma \Gamma}=\frac{1}{4} \sqrt{(-1)^{D-1} g} G^{(\mu \nu, \rho)(\alpha \beta, \sigma)} g_{\mu \nu, \rho} g_{\alpha \beta, \sigma}
$$

where

$$
G^{(\mu \nu, \rho)(\alpha \beta, \sigma)}=\left(g^{\mu \nu} g^{\alpha \beta}-g^{\mu \alpha} g^{\nu \beta}\right) g^{\rho \sigma}+2\left(g^{\mu \alpha} g^{\beta \rho}-g^{\mu \rho} g^{\alpha \beta}\right) g^{\nu \sigma} .
$$

(Eq. (77) in [10] has the opposite sign, but we keep convention of [6].)

In $2 D$, there are no contributions with two temporal derivatives. This immediately follows from Eq.(9) of [10]:

$$
g_{i k, 0} g_{m l, 0} G^{(i k, 0)(m l, 0)}=g_{i k, 0} g_{m l, 0} g^{00}\left(e^{i m} e^{k l}-e^{i k} e^{m l}\right)
$$

where $e^{i k}=g^{i k}-\frac{g^{0 i} g^{0 k}}{g^{00}}$. (Latin indices indicate spatial components.)

Similarly, the contributions to (6) that have two spatial derivatives vanish in $2 D$,

$$
\left[g_{\mu \nu, k} g_{\alpha \beta, m} G^{(\mu \nu, k)(\alpha \beta, m)}\right]_{D=2}=0 .
$$

However, some cross-terms with both spatial and temporal derivatives do not cancel and can be presented in the following, "semi-covariant", form

$$
L_{\Gamma \Gamma}=\frac{1}{2} \sqrt{-g} e^{11} g^{00} g^{\alpha \beta}\left(g_{0 \alpha, 1} g_{\beta 1,0}-g_{0 \alpha, 0} g_{\beta 1,1}\right) .
$$


It is not difficult to perform variation of (10) with respect to the metric $g_{\alpha \beta}$. This results in trivial equations of motion, consistent with what is a well-known result. However (10) is not equal to zero identically and cannot be put in the form of a total derivative, contrary to the generally held belief, as it was written in [8] and in many other articles including our own [11] where we thoughtlessly repeated this. Unfortunately, we also refer to [6] as a proof of it, which is entirely our mistake (we would like to note that L.D. Landau and E.M. Lifshits do provided a proof of the triviality of Einstein equations in $2 D$ but they had never made the conjecture that triviality of the equations of motion is equivalent to the Lagrangian being a total divergence). Of course, (10) can be made equal to zero by choice of a particular coordinate system or a subset of coordinate systems, but this contradicts the Dirac procedure [12] for passing to a Hamiltonian formulation for gauge theories. The main tenet of this procedure is to avoid any reference to a particular coordinate system, because when using a Hamiltonian general formulation, gauge transfromations can be restored from a knowledge of the first class constraints which should be independent of any choice of coordinate system.

One simple example in which (10) vanishes is the synchronous coordinate system (or "synchronous gauge" [5]) in which

$$
g_{0 k}=0, g_{00}=1
$$

and time lines are normal to the hypersurfaces $x_{0}=$ const (see Sec. 97 [6] ). Under condition (11), equation (10) is zero and $V_{, \alpha}^{\alpha}$ in (3) is equal to $2\left(\sqrt{-g_{11}}\right)_{, 0,0}$.

Similarly, the coordinate transfromations

$$
g_{\mu \nu}=e^{\phi} \eta_{\mu \nu}
$$

reduces (10) to zero and $V_{, \alpha}^{\alpha}$ becomes $\partial_{\mu} \partial^{\mu} \phi$ (i.e., see p. 304 of [13]).

In [4] the proof that $\sqrt{-g} R$ is a total divergence in Cartan variables was given using an orthonormal basis. Any choice of variables which leads to $g_{01}=0$ (and, consequently, $\left.g^{01}=0\right)$ makes (10) equal to zero. Not surprisingly, in such cases, the canonical formalism "breaks down" 3] and the action is meaningless [4]. If some reformulation of the EH action reduces the action to a total divergence in $2 D$, it means that general covariance in such formulation has been destroyed.

If we keep the action covariant (without specifying a coordinate system) then a canonical formulation of (10) can be performed (contrary to the conjecture of [3]). The Lagrangian 
(10) is very simple, and passing to a Hamiltonian form is straightforward since all terms are linear in velocities and introduction of momenta $\pi^{\alpha \beta}$ conjugate to metric tensor $g_{\alpha \beta}$ leads immediately to three primary constraints [12]

$$
\begin{aligned}
\phi^{00} & =\pi^{00}+\frac{1}{2} \sqrt{-g} g^{00} g^{11}\left(g^{00} g_{01,1}+g^{01} g_{11,1}\right), \\
\phi^{01} & =\pi^{01}-\frac{1}{4} \sqrt{-g} g^{00} g^{11}\left(g^{00} g_{00,1}-g^{11} g_{11,1}\right), \\
\phi^{11} & =\pi^{11}-\frac{1}{2} \sqrt{-g} g^{00} g^{11}\left(g^{01} g_{00,1}+g^{11} g_{01,1}\right) .
\end{aligned}
$$

The Hamiltonian is then just a linear combination of constraints (13)

$$
H=\lambda_{\alpha \beta} \phi^{\alpha \beta}
$$

Using the fundamental Poisson brackets (PB)

$$
\left\{g_{\alpha \beta}, \pi^{\mu \nu}\right\}=\frac{1}{2}\left(\delta_{\alpha}^{\mu} \delta_{\beta}^{\nu}+\delta_{\alpha}^{\nu} \delta_{\beta}^{\mu}\right)
$$

it is easy to demonstrate that each of the constraints has a vanishing PB with itself as expected because of the antisymmetric properties of the PB. (This is unlike the hypersurface deformation algebra of the ADM constraints [14].) This is actually obvious because in all constraints appearing in (13) there are no spatial derivatives of the components of $g_{\alpha \beta}$ which correspond to components of the conjujate momenta $\pi^{\alpha \beta}$. Moreover, the PB's among different constraints are also zero and the resulting algebra is quite trivial:

$$
\left\{\phi^{\alpha \beta}, \phi^{\mu \nu}\right\}=\frac{\delta \phi^{\alpha \beta}}{\delta g_{\rho \sigma}} \frac{\delta \phi^{\mu \nu}}{\delta \pi^{\rho \sigma}}-\frac{\delta \phi^{\mu \nu}}{\delta g_{\rho \sigma}} \frac{\delta \phi^{\alpha \beta}}{\delta \pi^{\rho \sigma}}=0 .
$$

From (14, 16) it is obvious that there are no secondary constraints. The Dirac procedure is closed; standard counting of degrees of freedom leads to zero as there are three first class (FC) constraints corresponding to the three independent components of the metric tensor.

From these three primary $\mathrm{FC}$ constraints we can construct the gauge generator $G(\epsilon)$ using the Castellani procedure [15]. In this case we simply get

$$
G(\epsilon)=\int d x \epsilon_{\alpha \beta} \phi^{\alpha \beta}
$$

where the $\epsilon_{\alpha \beta}$ are gauge parameters.

The gauge transformations of $g_{\alpha \beta}$ can be found from $\delta g_{\alpha \beta}=\left\{g_{\alpha \beta}, G(\epsilon)\right\}$ giving,

$$
\delta g_{\alpha \beta}=\epsilon_{\alpha \beta}
$$


This is expected as the equations of motion are trivial equations which are valid for any $g_{\alpha \beta}$.

It is easy to check that these gauge transformations leave $S_{E H}$ invariant up to a surface term, because the variation of $L_{\Gamma \Gamma}$ is:

$$
\delta L_{\Gamma \Gamma}=\frac{1}{2} \partial_{\gamma}\left[\sqrt{-g}\left(g^{\rho \sigma} g^{\beta \gamma}-g^{\rho \gamma} g^{\beta \sigma}\right) g^{\alpha \mu} g_{\mu \rho, \sigma} \epsilon_{\alpha \beta}\right] .
$$

We have demonstrated that the statements that $2 D \mathrm{EH}$ action is meaningless and that canonical procedure for it breaks down are not in fact correct and are just a consequence of not following canonical procedure by having made the choice of some "priviledged" coordinate system or a family of such systems including "slicing of spacetime". (According to Hawking 16] this also contradicts to the spirit of GR.) Does any meaning exist in "canonical" formulations which results depend on a particular coordinate system and in some dimensions cannot be even formulated? Of course, it is possible to use a specific coordinate system to study particular classical solutions of the Einstein field equations, but it is inappropriate to make such a choice when quantizing GR, as quantum fluctuations are not restricted to a particular choice of coordinates. If the canonical procedure is performed in a fixed coordinate system, we cannot guarantee that it will reproduce the same invariance as that present in the original action. In $2 D$ the use of a particular system does not even allow one to perform a canonical procedure. If we want to ensure that the Quantum Gravity is consistent with GR we have to retain the principle of general covariance when applying canonical procedure and not destroying general covariance from the beginning. The simple example of the EH action in $2 D$ shows the importance of keeping general covariance when using the canonical procedure.

Invariance of the Lagrangian up to a surface term is different from the exact invariance occuring in ordinary gauge theories and one needs to impose an extra condition on behavior of gauge parameters at infinity as, for example, in the case of invariance of the GammaGamma part of the EH action under linearized coordinate transformations (see p. 272 of 6]). In particular, we are not aware of any canonical formulation of the $2 D \mathrm{EH}$ action that restores these transformations. The use of the ADM formulation [17] for the $2 D \mathrm{EH}$ action leads to the unphysical result of there being negative degrees of freedom [3]; i.e. it is an overconstrained system. Contrary, our (18) is the result of canonical procedure for the non-divergence part of the $2 D \mathrm{EH}$ action.

However, we think that invariance up to a surface term as given by (19) could be the 
result of another deviation from general covariance, not peculiarity of EH action. The reason for this is the elimination of a total divergence in our consideration and actually taking only

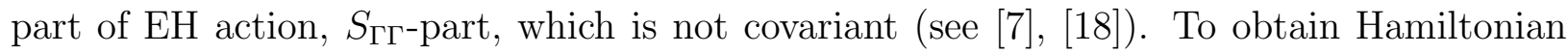
formulation which can lead to gauge transfromations of the same transforming power as general coordinate transformations of the EH action (not only $S_{\Gamma \Gamma}$ ) we have to keep the effect of all terms in the course of canonical procedure (for discussion of this point see 19]). In higher dimensions one possibility is to use the equivalent first order affine-metric formulation of Einstein [20], which, however, cannot be used in 2D, as affine connections in

this case cannot be found in terms of the metric tensor [2]. This is also true for its particular combination used in [19] and, actually, for any linear combination of them.

So, a first order formulation different from the affine-metric one has to be used in a canonical approach to the $2 D \mathrm{EH}$ action. There exists a variety of first order formulations (non-symplectic) that can be constructed keeping equivalence with the second order form. The question is whether one of them can give consistent Hamiltonian formulation and what gauge transformations it will produce.

We can also try to capture the effect of all terms in the EH action performing the canonical procedure by considering the EH action as a theory with higher derivatives and using Ostrogradsky method [21] with modifications appropriate for singular systems [22, 23]. (The first attempt to apply this to the full EH action is due to Dutt and Dresden [24].)

We would like to conclude by referring to a conjecture of Dirac [12]: "I (Dirac) feel that there will always be something missing from them (non-Hamiltonian methods) which we can only get by working from a Hamiltonian". This article provides an example that is an illustration of this conjecture; despite triviality of equations of motion, the Hamiltonian formulation allows one to find a gauge transformation and the corresponding invariance of the action.

\section{ACKNOWLEDGMENTS}

The authors are greateful to D.G.C. McKeon for discussions and reading the manuscript. We thank E.V. Gorbar for reading of the final version of manuscript, S.B. Gryb for discussions, D.V. Prokopiev and E.Z. Prokopieva for useful suggestions and R. Corless for 
inspiration and support.

[1] J. Polchinski, Nucl. Phys. B324 (1989) 123.

[2] J. Gegenberg, P.F. Kelly, R.B. Mann and D. Vincent, Phys. Rev. D37 (1988) 3463.

[3] E. Martinec, Phys. Rev. D30 (1984) 1198.

[4] T. Strobl, hep-th/0011240.

[5] T. Banks and L. Susskind, Int. J. Theor. Phys. 23 (1984) 475.

[6] L.D. Landau and E.M. Lifshits, The Classical Theory of Fields, 4th edition (Pergamon Press, Oxford, 1975).

[7] M. Carmeli, Classical Fields: General Relativity and Gauge Theory, (Wiley, New York, 1982).

[8] R. Jackiw, Nucl. Phys B252 (1985) 343.

[9] F.A.E. Pirani, A. Schild and R. Skinner, Phys. Rev. 87 (1952) 452.

[10] P.A.M. Dirac, Proc. Royal Soc., London 246 (1958) 333.

[11] N. Kiriushcheva, S.V. Kuzmin and D.G.C. McKeon, Mod. Phys. Lett. A20 (2005), 1895; ibid. 1961.

[12] P.A.M. Dirac, Lectures on Quantum Mechanics, (Yeshiva University, New York, 1964).

[13] M.B. Green, J.H. Schwarz, E. Witten, Supersrting Theory, Vol.2 (Cambridge University Press, Cambridge, 1986).

[14] T. Thiemann, gr-qc/0110034.

[15] L. Castellani, Ann. Phys. 142 (1982) 357.

[16] S.W. Hawking, The path-integral approach to quantum gravity, in: General Relativity. An Einstein centenary survey. Eds. S.W. Hawking and W. Israel (Cambridge University Press, 1979), 746.

[17] R. Arnowitt, S. Deser, and C.W. Misner, The dynamics of General Relativity, in: Gravitation: An Introduction to Current Research ed. by L. Witten, (Wiley, New York, 1962) 227.

[18] F.A.E. Pirani and A. Schild, Phys. Rev. 79 (1950) 986.

[19] N. Kiriushcheva and S.V. Kuzmin, hep-th/0507074 (to appear in Annals of Physics).

[20] A. Einstein, Sitzungsber.preuss. Akad. Wiss., phys.-math. K1, (1925) 414 and The complete collection of scientific papers (Nauka, Moskow, 1966), v.2, p.171.

[21] M. Ostrogradsky, Memoires de l'Academie Imperiale des Science de Saint-Petersbourg, IV 
(1850) 385.

[22] D.M. Gitman and I.V. Tyutin, Izvestiya Vuz. Fizika, 26 (1983) 61; Sov. Phys. J. (1984) 730.

[23] D.M. Gitman and I.V. Tyutin, Quantization of Fields with Constraints (Springer-Verlag, Berlin, 1990).

[24] S.K. Dutt and M. Dresden, Pure gravity as a constrainted second-order system, Preprint ITP-SB-86-32. 